\title{
Sitzung der Berliner ophthalmologischen Gesellschaft
}

vom 26. 3. 1903

Vorsitzender: Herr du Bois Reymond. Schriftführer: Herr Wertheim.

Herr du Bois Reymond beantragt eine Veränderung der Statutenordnung, nachdem die Zahl der Mitglieder von ursprünglich 16 nunmehr durch Eintritt der meisten Berliner Ophthalmologen auf 76 gestiegen ist. - Der Antrag wird genehmigt und in Uebereinstimmung mit dem auf Reisen befindlichen bisherigen ersten Vorsitzenden, Herrn Geh. Rat Hirschberg, schlägt Herr du Bois Reymond die Wahl des Herrn Geh. Rat v. Michel zum 1. Vorsitzenden vor. Herr Geh. Rat v. Michel wird per acclamationem zum 1. Vorsitzenden der Berliner ophthalmologischen Gesellschaft gewählt, ebenso Herr Geh. Rat Hirschberg zum stellvertretenden, Herr Prof. Greeff zum dritten Vorsitzenden. Die Herren Wertheim und du Bois Reymond werden als Schriftführer wiederum, Herr Schöler jun. als dritter Schriftführer neu gewählt.

Herr Fehr stellt eine 62jährige Prau mit einem Tumor auf derIrisvorderfläche vor, die bereits seit 13 Jahren sich in BeobachtungZeitschrift f. Augenheilkuude. Bd. IX. Heft i. 22 330

Offene Korrespondenz.

befindet. Per exclusionem nimmt Fehr ein sehr langsarn wachseades Sarcom an, und scbliesst Melanom sowie Granulationsgeschwulst aus. Entzündungen bestehen nicbt.

Herr Schöler jun. stellt einen Knaben mit linksseitiger Abduceus-parese vor, bei dem dann Kopfschmerzen, Erbrechen, Schwindel, am 7. Januar d. J. rechtsseitige Facialisparese, danach linksseitige totale Parese des Rumpfes eintrat. Die Sprache ist jetzt auch bereits gåz unverständlich: es bestebt keine Stauungspapille. Schöler erklärt sich für eine Ponserkrankung und plaidiert für einen Tumor (Gliom oder Tuberkel).

Herr Oppenbeimer demonstriert einen Knaben mit Molluscum contagiosum besonders am Intermaginalsaum.

Herr Hoffmann stellt eine 29 jäbrig,e Prau mit ausgebreitetem Melanom der Conjunctiva des línken Auges vor. S. = r, $/ 5.0 . \mathrm{n}$.

Herr Salomonsohn berichtet tiber seine Erfahrungen mit Scopolamin, das er in stärkerer Dosis bei chronischer Iritis seit einiger Zeit anwendet. Statt der üblichen 1ii proc. Lösung giebt $\mathrm{S}$ jetzt oft eine 3 proc. (!) Lösung, trotzdem dabei meist leichte Intoxicationserscheinungen auftreten. Des Weiteren empfiehlt S. das Yo him bin in 1 proc. Lösung als Anaestheticum. Die Anaesthesie dauert ca. 1/2 Stuude, die Accommodation wird nicbt beeinflusst. Störend ist freilich die starke Гlyperämie.

Pollack.

Offene Korrespondenz.

I. Im letzten Hefte dieser Zeitschrift ist eine Arbeit von Hála über dieCorynebakterien zum Abschluss gebracht worden welche nochmals der heutcwohl ziemlich allgemein geteilten Anschauung Ausdruck verleiht 
dass durch-greifende Unterschiede zwischen Xerose- Pseudodiphtherie- und Diph-tberiebacillen nicht bestehen. Auch die früher das alleinige Unterseheidungs-merkmal liefernde Virulenzprüfung ergab bei entsprechender Versuchs-anordnuug Resultate welche diese Unterschiede nur noch mehr zu verwischengeeignet sind und so kommtHála auf Grund eingehenđler Untersuchung zueinem Resultate welches im wesentlichen nur eine ßestätigung einer Arbeitist die ich bereits 1896 in den Sitzungsberichten der niederrheinischenGesellschaft für Natur- und Heilkunde (vollständig abgedruckt in derDeutschen medizinischen $\Lambda$ V"ochenschrift 1897) veröffentlicht habe und derenResultate in dem Werke von Flügge „Die Mikroorganismen” durch Kruseauf S. 478 des II. Bandes eine eingebende Besprechung erfahren haben. DerUmstand dass Hála in seiner sonst ziemlich vollständigen Litteratur-zusammenstellung gerade eine der ersten Arbeiten

die sich das vergleichendeStudium dieser Mikroorganismen zur Aufgabe stellte vollkommen übersehenhat giebt mir Veranlassung an dieser Stelle nochmals darauf hinzuweisen, dass ich schon damals bei den Pseudodiphthèriebacillen eine Art leichterVirulenz bei der subperitonealen Einverleibung bei Meerschweinchen kon-statieren konnte. Auf diese Arbeit nochmals hinznweisen erscheint mirauch aus dem Grunde wünschenswert weil sie auch bei anderen Autoren, die auf diesem Gebieto gearbeitet haben bereits in Vergessenheit geratenzu sein scheint. prof_Peters.RostocL 